\title{
Bayesian strategies for the reliability assessment of heritage structures
}

\author{
P. Croce ${ }^{1} \&$ F. Marsili ${ }^{1,2}$ \\ ${ }^{I}$ Department of Civil and Industrial Engineering, \\ Structural Division University of Pisa, Italy \\ ${ }^{2}$ University of Braunschweig, Germany
}

\begin{abstract}
The reliability assessment of heritage structures is becoming an increasingly important and frequent engineering task. Probabilistic methods and Bayesian updating techniques are often combined to obtain realistic estimates, also aiming to target the best the possible interventions. Moreover, several factors often contribute to complicating the probabilistic approach, including uncertainties about the knowledge of the mechanical properties of materials, due to a lack of quantitative data from the structure. In this paper, a strategy to perform Bayesian updating used in assessing heritage structures, based on qualitative a posteriori judgment, is proposed, where the probability distribution function for the material properties are updated on the basis of the information available on similar structures. A case study concerning a relevant historical building is also presented. Keywords: heritage structures, reliability assessment, Bayesian analysis.
\end{abstract}

\section{Introduction: a classification of existing structures}

For many centuries, structural and architectural features were both embraced by a single holistic design process; an adequate structural design was assured by the architectural type and order, together with regulator alignments and proportional rules based on the empirical evidence of successfully performing structures. Building techniques were generally codified on empirical basis, even if adapted to strong local traditions, while the materials were often of local origin. The most of the structures were made of brick or stone masonry, sometime combined with timber elements, where greater tensile or bending capacity was called for [1]. 
Clearly, as structural dimensioning adopting empirical formula can be sensibly different from that we could determine today, historical structures, even not affected by structural failures, largely exhibit lack of reliability if inspected in the light of modern codes.

A time of changes was represented by the emergence of modern science during the early modern period, when developments in applied mathematics, physics and chemistry allowed to give more and more sound theoretical bases to the design and constructing process, separating the structural aspects of the building from the architectonical canons and its visual appearance.

Firstly, Galileo rejected the use of both the architectural types and the proportional design rules, revealing that geometric similitude does not imply mechanical similitude [2], subsequently Hooke laid the basis of the theory of elasticity and finally Navier and De Saint-Venant developed it, making possible the application of mathematical sciences to building engineering.

At the same time, the outcomes of the Industrial Revolution made available other advanced building materials, so that the choice, originally limited to timber and masonry, widened considerably, while easier transports and world-wide trade allowed the phenomenon to become global. It began with the large-scale production first of iron and cast iron and then of steel; next came Portland cement followed by the highly versatile composite material, reinforced concrete. A wider choice of construction material led to a sudden development of new structural types, often resulting ineffective or unsuccessful. In fact, from one side the methods for calculating even simple indeterminate structures were still in an early stage, from the other side poor detailing and poor workmanship were the result of the unavoidable lack of knowledge and experience in new materials. Progress in building design and construction was therefore slow and often attributed to analogical methods and trial-and-error processes.

The $20^{\text {th }}$ century represented a new dawn for structural engineering, with the development of codes and standards aimed at giving common basis for design, so assuring the achievement of target reliability level. Early codes adopted design procedure based on the precepts of allowable stress design: the concept of an arbitrary Factor of Safety was suggested by Freudenthal, and the possibility of rational probability-based design began slowly to emerge.

The first attempt to draw Limit State codes, introducing separate partial safety factors on loads and materials, dates around 1930-1935, but it was during the 1970 s that limit state concepts became mature. The Limit State design was not just a change in calculation format: the intention was that variations in loads, materials and member strengths would be statistically analysed and probability theory used to calculate more rational design values. The underlying aim was to produce structures with approximately uniform reliability against exceeding a limit state. However, because of the lack of relevant statistical data and the need to preserve accustomed tradition, partial factors were still largely based on past practice and subjective judgments.

Nowadays, the principle according to which a structure should be designed to have appropriate degrees of reliability in regard to ultimate and serviceability limit states represents a basis of the Eurocodes [3], that, together with durability and 
robustness requirements, give guidance on design methods based on reliability analysis, permitting also their application for special design.

According to the brief evolution of construction and design outlined above, it is possible to propose a classification of existing structures in terms of design approach, materials and structural system:

1. Ancient structures (until late $18^{\text {th }}$ century): mainly made of masonry and wood, designed according to architectural canons and empirical formulae;

2. Modern structures ( $19^{\text {th }}$ century): built with iron, cast iron, steel and reinforced concrete, designed according to theory of elasticity principles and a trial-and-error processes, characterised by structural systems generally composed by trusses and frames;

3. Contemporary structures (from the $20^{\text {th }}$ century until today): mainly built with steel and reinforced concrete, designed according to Codes and Standards that implement a set of partial factors, taking into account all the uncertainty involved in the design and construction process.

Due to environmental, economic and socio-political reasons, significance and field of application of existing structures assessments' extend rapidly, also in view of preservation of cultural heritage. Despite assessment of existing buildings and design of new buildings differ in many aspects, in them basic variables can be handled similarly through partial factors. On the other hand, considering that an increase of the reliability level is usually more costly for an existing structure than for a new one, which is still in the design phase, in the assessment of existing structures a reliability level lower than that required for new structures is generally accepted, in order to avoid unnecessary expensive interventions, that, in case of heritage buildings, also entail a loss of the related and predominant cultural value. In order to properly handle all the uncertainties affecting the assessment process, probabilistic methods are often invoked; in this way it is also possible to perform more 'realistic' reliability assessment, based on the actual characteristics of the structure.

In the following, a strategy to perform a Bayesian updating in assessing heritage structures, based on qualitative a posteriori judgment is proposed, where probability distribution function for material properties are updated on the base of information available on similar structures.

\section{Probabilistic methods for the reliability assessment of existing structures}

A probabilistic reliability assessment is based on the following steps [4]:

1. identification of the relevant ultimate and serviceability limit states;

2. identification of the failure modes leading to the limit state, e.g. ultimate strength, yielding, bending, buckling, fatigue, deformation, vibration;

3. identification of the basic variables that govern the failure mode, e.g. dimensions of structural elements, intensity and nature of actions, material properties, model uncertainties and internal forces; 
4. definition of probability distribution function $(p d f)$ for the basic variables that govern the limit state;

5. definition of appropriate limit state functions expressing in the considered cases the fundamental requirement of the theory of structural reliability:

$$
E<R
$$

where the resistance $R$ and the action effect $E$ are suitably distributed random variables. This condition leads to the fundamental forms of the limit state functions:

$$
G=R-E=0 \text { or } Z=R / E=1
$$

where $G$ is the safety margin and $Z$ the safety factor.

The essential object of the reliability theory is to assess the probability of failure:

$$
p_{f}=P[G<0]=P[R-E<0] .
$$

The failure probability $p_{f}$ in structural engineering can be obtained using a simplified approach, based on the estimation of the reliability index $\beta$, which is a function of $p_{f}$,

$$
\beta=-\Phi^{-1}\left(p_{f}\right)
$$

6. verification of the structural reliability: the goal for reliability analysis is to document that the target reliability reflecting the 'accepted' level of risk in terms of possible failure consequences in a given reference time period is achieved. The following verification formats are considered:

$$
p_{f}<p_{d} \text { or, equivalently, } \beta>\beta_{t}
$$

where the target reliability is represented by $p_{d}$ or $\beta_{t}$.

An assessment carried out with probabilistic methods is validated by the fact that the structure to be assessed has been designed according to codes or methodologies already based on the application of probabilistic reliability analysis, such as the Partial Factor Method adopted by current codes. In this case, the Bayesian analysis is often applied in order to update the information from the original design with the result of a test and inspection carried out on the actual structure and a defining of the actualized $p d f$ s.

\section{Bayesian analysis in the reliability assessment of existing structures}

The aleatoric uncertainties accompanying the design and erection phases as well as the design life of contemporary structures are usually taken into account through model uncertainty factors that, together with the representative value for action and resistance and the related partial factors, are clearly given by the codes. Once the structure completed, it shifts from an ideal entity to a physical object, and uncertainties changes from aleatoric to epistemic. Therefore, an existing structure can be suitably investigated, and the imperfect knowledge about its properties (material characteristics, actions, structural model, geometry and so on) can be actualized. The framework for doing this is the Bayesian statistic, which uses Bayes Theorem [4]. According to Bayesian analysis, the definition of $p d f$ s of basic variables can be improved as follows: 
1. definition of a prior $p d f f_{X}{ }^{\prime}(x)$ for the random variable $X$ based on the original knowledge, assuming that one or more parameter of the distribution, e.g. the mean value or the standard deviation of $X$, are uncertain themselves and described by a prior $p d f f_{Q}{ }^{\prime}(q)$;

2. collection of a set of observations of $X: \hat{x}=x_{1}, x_{2}, \ldots, x_{n}$;

3. definition of the a posterior or updated $p d f f_{Q}{ }^{\prime \prime}(q)$ of $X$ :

$$
f_{Q}^{\prime \prime}(q \mid \hat{x})=\frac{f_{Q^{\prime}}(q) L(q \mid \hat{x})}{\int_{-\infty}^{+\infty} f_{Q^{\prime}}(q) L(q \mid \hat{x}) d q}
$$

where $L(q \mid \hat{x})$ is the likelihood of the observations $\hat{x}$; the observation $\hat{x}$ can also be used to directly update the $p d f$ of $X$, so obtaining:

$$
f_{X}^{\prime \prime}(x)=\int_{-\infty}^{+\infty} f_{X}(x \mid q) f_{Q}^{\prime \prime}(q \mid \hat{x}) d q
$$

The updating of single variables will result, obviously, in the updating of the probability of failure. Therefore, the concept can be illustrated in general way for the updating of the $p d f$ of $G$. If $f_{G}^{\prime}($.$) represents the a priori (conditional) p d f$ of $G$, and $f_{v}($.$) represents the (conditional) p d f$ based on new (measured) data, then a posteriori or updated $p d f f_{G}^{\prime \prime}($.$) can be derived, as shown in fig. 1$.

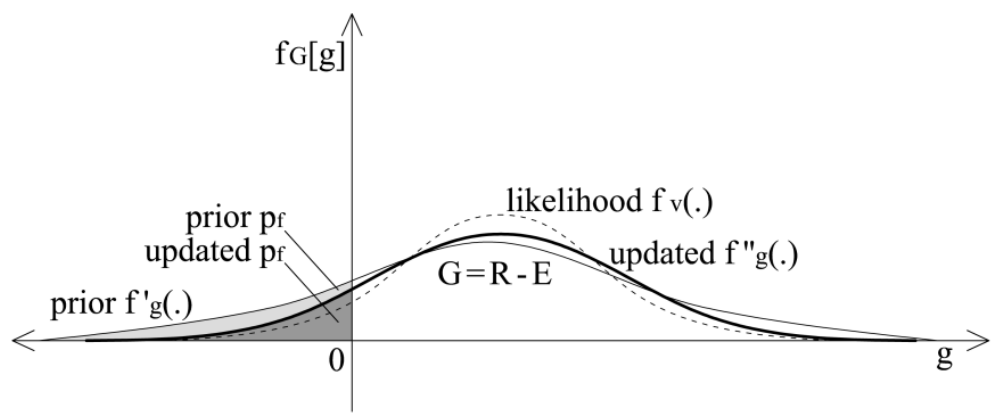

Figure 1: Updating of $p_{f}$.

\section{Limits of the Bayesian analysis of historical structures}

\subsection{Difficulties in collecting prior information}

While for contemporary structures it is possible to go back to the original drawings and calculation, as well as standard and codes applied when the structure was designed, and for modern structures it is possible to refer to original guidelines and original companies, as well as engineering textbooks and publications on professional journals and newspapers, poor information are generally available regarding ancient structures built till the $18^{\text {th }}$ century, in absence of any formal design.

An exception is often represented by significant and monumental buildings that played an important role in the politics, social interactions, and economics of the day, for which historical records exist [5]. In this case, significant source of information are archive studies, which allow to collect, for instance, contracts and 
specifications with relevant project drawings for the construction, contracts and bills referring to production, sale and purchase of building materials, judgements or court deeds regarding controversies in the building sector, and so forth. Other relevant sources are treatises, manuals and technical literature published during the $18^{\text {th }}$ and $19^{\text {th }}$ century, collecting in written form the existing empirical knowledge about building practice.

For vernacular architecture information if even scarcer. The available knowledge is represented by contemporary studies and researches about material, construction techniques, and structural typologies typical of a certain age or geographical area. This kind of information, derived from the literature or from available modern databases [6], could be used as a prior knowledge of the structure to be assessed, based on properties of similar buildings. A deep knowledge of construction techniques commonly used in that period may help in identifying the original structural condition and appearance. For building built on empirical bases, the original design is difficult to define and the recognition of the actual structural behaviour is often vague, but a global analysis of the original structure, although rough and approximated, may help in explaining identified damage scenarios and understanding causes of degradation. Moreover, it should be emphasized that old buildings are hardly structurally sound.

On the contrary, buildings designed with a formal approach can be analysed simulating the original design, i.e. reproducing the design process according to methodologies and codes commonly used when the structure was designed [7], in order to improve or enlarge the knowledge about dimensions and mechanical performances of structural members.

\subsection{Difficulties in investigating the structure}

Investigations on existing buildings are often devoted to recognize the actual structural scheme, relevant actions, material characteristics, geometry and model uncertainties. The outcomes of the inspections can be qualitative, if they are given in terms of qualitative judgments, or quantitative, if they are represented by set of values of relevant parameters. Usually preliminary investigation are qualitative, while, when a more detailed evaluation is required, it could be necessary to plan some semi-destructive or even destructive test campaign.

The belief that intrusive inspections and tests improve the understanding of the structure is put in doubt when the object of the studies is represented by an ancient structure.

First of all, an existing structure is much more than a structure just built, since it is also the result of the time passage; this is especially true for historical building, where aging, deterioration, environmental influences and human interventions have changed the actual reliability level during the centuries. Modification and degradation usually have a great impact on structural integrity, leading to weak points and structural defects that are seldom documented and not easy detectable. Disregarding those anomalies entails a misunderstanding of the structural scheme that could lead to unacceptable errors in reliability assessment [8]. Recognition of structural alteration is therefore conditio sine qua non for dependable reliability assessments. 
Although, destructive investigation techniques can result very helpful to determine the most relevant mechanical parameters and to identify structural hidden features, in case of ancient structures they often entail the loss of the cultural value embodied in the building, so that they should be avoided.

The application of non-destructive techniques (NDTs) to ancient structures can be frustrated by several factors, like the high non homogeneity of the materials, the differences in masonry typologies, the harmonisation and interpretation of the results of each single technique [9]. More reliable results can be achieved, combining several NDT, but their outcomes will be mainly qualitative, unless more precise calibrations are performed on experimental basis, like in "lucky" case, when destructive test campaign can be carried out on portion of structures addressed to demolition, or on samples collected in situ (and eventually recomposed in laboratory) after the collapse of a part of the structure. Obviously, setup of suitable methodologies to extend available experimental results to similar and coeval structures could represent a significant progress in the field.

\section{Updating of masonry characteristics: a possible course of action}

\subsection{General considerations}

Masonry is a composite material, whose mechanical characteristics and structural performances depend on the nature of the components and on the adopted construction technique. Usually, masonries with different mechanical and physical behaviors can be originated depending on the following factors:

- physical, chemical and mechanical characteristics of the components;

- texture and dimensions of elements;

- dimensions of cross section.

The question that arises is: how is it possible to derive an accurate probabilistic model for the mechanical characteristics, when no information from the original design is available, the material is highly non-homogeneous and samples have to be numerous and non-destructive?

A possible course of action is represented by the fact that the mechanical behavior of the masonry is mostly governed, rather than by the properties of its components, by the texture and the characteristics of the cross section; features that can be easily assessed by visual inspection, or with the implementation of NDTs, as suggested by several codes.

For instance, in the Italian Code [10], a general classification of recurrent masonry types, based on their external texture, is proposed. For each type, minimum and maximum values, corresponding to $16 \%$ and $84 \%$ fractiles of the corresponding $p d f$, are suggested for the most relevant mechanical properties [11]. Of course, a prior $p d f f_{Z}{ }^{\prime}$ could be defined for the mechanical property $Z$, according to tabulated values, but these prior $p d f \mathrm{~s}$ are clearly very scattered, since they must cover a wide range of real situations. The question that arises now is: is it possible to reduce the scatter through qualitative judgments and visual inspections, without performing destructive tests? 
The basic idea is that, as similar masonries are characterized by comparable mechanical parameters, a posterior knowledge can be achieved adopting the results of in situ or laboratory destructive tests, previously performed on masonry specimens similar, in terms of dimensions and external texture, to the one to be assessed.

Like the previous case considered in $\S 3$, a prior $p d f f_{X}{ }^{\prime}$ that describes the variability of the parameter of interest $X$ can be established. But, in this circumstance, test results about the $X$ parameter on similar masonry are available, so that $f_{X}{ }^{\prime}$ can be updated with the sample of observation $X$ performing a Bayesian analysis. What we get is a posterior $p d f f_{X}{ }^{\prime \prime}$, hopefully less scattered that the prior one because some uncertainties have been reduced.

Referring to the algebra of the distributions, the effect of the updating can be expressed with a couple of corrective coefficients that 'adjust' the parameters of the prior distribution and bring to the updated values.

The procedure is described in the following. For the sake of simplicity, we refer to Aitchison and Brown [12] for the chosen nomenclature.

Let the prior $p d f$ lognormal,

$$
f_{X}^{\prime}=\operatorname{LN}\left(\mu_{X}^{\prime}, \sigma_{X}^{\prime 2}\right)
$$

the posterior or updated $p d f$ can be written

$$
f_{X}^{\prime \prime}=\operatorname{LN}\left(\mu^{\prime \prime}{ }_{X}, \sigma^{\prime \prime 2}\right) \text {. }
$$

Since it holds:

$$
f_{X}: \operatorname{LN}\left(\mu_{X}, \sigma_{X}^{2}\right) \rightarrow e^{a} f_{X}^{b}: \operatorname{LN}\left(\mathrm{a}+\mathrm{b} \mu_{X}, b^{2} \sigma_{X}^{2}\right),
$$

it is possible to define $a, b$

$$
f_{X}{ }^{\prime \prime}=e^{a} f_{X}{ }^{b}: \operatorname{LN}\left(\mathrm{a}+\mathrm{b} \mu_{X}^{\prime}, b^{2} \sigma_{X}^{\prime 2}\right)
$$

solving the following system of equation:

$$
\left\{\begin{array}{c}
\mu_{X}^{\prime \prime}=\mathrm{a}+\mathrm{b} \mu_{X}^{\prime} \\
\sigma^{\prime \prime 2}{ }_{X}=b^{2} \sigma^{\prime 2}{ }_{X}
\end{array}\right.
$$

Because of the similarities between the masonry to assess and the tested masonry, it is possible to use the corrective coefficients to 'adjust' the parameters of the prior $p d f$ of the masonry to assess.

Assuming that

$$
f_{Z}^{\prime}=\mathrm{LN}\left(\mu_{Z}^{\prime}, \sigma_{Z}^{\prime 2}\right)
$$

is the prior $p d f$, we obtain the 'adjusted' $p d f$ in the form:

$$
f_{Z}{ }^{*}=e^{a} f_{Z}{ }^{\prime b}: \operatorname{LN}\left(\mathrm{a}+\mathrm{b} \mu_{Z}^{\prime}, b^{2}{\sigma^{\prime}}^{2}\right) \text {. }
$$

In this way the updating can be performed by visual inspection: the $p d f$ for basic variables of masonry characteristics is again refined through an engineering judgement, but supported by more objective and sound bases.

A practical application of this procedure to the masonry compressive strength of a historical structure is presented in the following example.

\subsection{Example: updating of the compressive strength of a masonry wall}

The Medicean Aqueduct of Pisa (Italy) is a masonry water work built in the early $17^{\text {th }}$ century; in view of its preservation, a reliability assessment is performed applying probabilistic methods, requiring the probabilistic description of the masonry compressive strength. 
A preliminary investigation is carried out, aimed at defining the general characteristic of the masonry. Attention is especially focused on visible features: the wall has a massive depth of $1.20 \mathrm{~m}$; both the external layer and the inner core of the masonry are made of irregular stones; the cross section is regularized by thin layer of bricks; mortar appears in good conditions. Considering the age of the structure, it is possible to affirm that masonry has a good quality (fig. 2). The structure can be classified as undressed stone masonry with regular texture. For the compressive strength of this kind of masonry, the Italian Code orders a minimum value of $2.60 \mathrm{~N} / \mathrm{mm}^{2}$ and a maximum value of $3.80 \mathrm{~N} / \mathrm{mm}^{2}$. However, if the cross section is wide, it is suggested to decrease those values of the $20 \%$. Therefore the prior probabilistic model for the compressive strength is represented by a log-normal $p d f f_{m, Z}{ }^{\prime}$ characterized by $\alpha_{Z}^{\prime}=2.50 \mathrm{~N} / \mathrm{mm}^{2}$ and $\beta^{\prime}{ }_{Z}=$ $0.505 \mathrm{~N} / \mathrm{mm}^{2}$.

An experimental investigation aimed at characterizing stone masonry walls with regular texture has been carried out in [13] on masonry specimens, built in the laboratory using stones and mortar typical of historical structures (fig. 2), classified, according to the Italian Code as 'undressed stone masonry'. The depth of cross section, composed by two vertical leaves of stones placed side by side and occasionally connected by large elements arranged transversely along the wall length, was $0.32 \mathrm{~m}$. According to the Italian Codes, the prior probabilistic model for the compressive strength $f_{m, X}{ }^{\prime}$ is represented by a log-normal $p d f$ characterized by $\alpha_{X}^{\prime}=3.20 \mathrm{~N} / \mathrm{mm}^{2}$ and $\beta_{X}^{\prime}=0.613 \mathrm{~N} / \mathrm{mm}^{2}$. Results of vertical compression tests performed on 6 specimens were: $\widehat{f_{m}}=$ $(3.14,3.09,3.76,3.18,3.13,3.36) \mathrm{N} / \mathrm{mm}^{2}$. The sample $p d f$ is still log-normal and characterized by $\alpha_{X}=3.278 \mathrm{~N} / \mathrm{mm}^{2}$ and $\beta_{X}=24.7 \mathrm{~N} / \mathrm{mm}^{2}$.

Applying the Bayesian updating techniques, the prior distribution has been updated considering test results. The posterior $p d f f_{m, X}{ }^{\prime \prime}$ is characterized by $\alpha_{X}{ }^{\prime \prime}=$ $326 \mathrm{~N} / \mathrm{mm}^{2}$ and $\beta^{\prime \prime}{ }_{X}=58.6 \mathrm{~N} / \mathrm{mm}^{2}$.

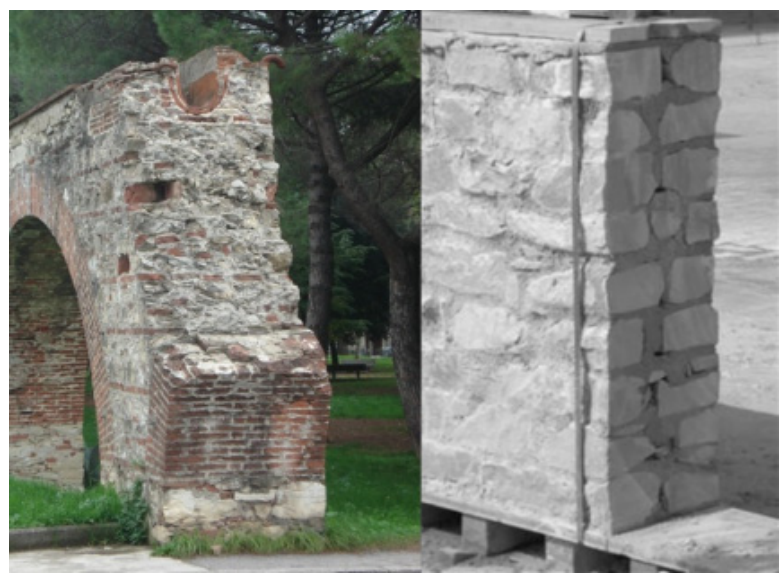

Figure 2: The masonry of the Medicean Aqueduct of Pisa, Italy (left) and a sample of the masonry wall tested in Magenes et al. [13] (right). 
A series of corrective coefficients that represent the effect of the Bayesian updating on the mean value and standard deviation of the prior $p d f f_{m, X}{ }^{\prime}$ are defined according to the previously mentioned procedure.

Considering that:

it holds:

$$
f^{\prime \prime}{ }_{m, X}=e^{a} f^{\prime}{ }_{m, X}^{b}
$$

$$
\left\{\begin{array}{l}
a=0.28 \\
b=0.91
\end{array}\right.
$$

The corrective coefficients can be applied in order to 'adjust' the prior $p d f f^{\prime}{ }_{m, Z}$ for the compressive strength to be assess:

and therefore:

$$
f_{m, Z}{ }^{*}=e^{0.28} f_{m, Z}^{\prime}{ }^{0.91}
$$

$$
\begin{gathered}
\mu_{Z}{ }^{*}=0.28+0.91 \alpha^{\prime}=5.517 \\
{\sigma_{Z}}^{2 *}=0.91^{2} \beta^{\prime 2}=0 ., 036
\end{gathered}
$$

that correspond to:

$$
\begin{aligned}
\alpha_{Z}^{*} & =2.535 \mathrm{~N} / \mathrm{mm}^{2} \\
\beta_{Z}^{*} & =0.488 \mathrm{~N} / \mathrm{mm}^{2} .
\end{aligned}
$$

The prior $p d f \mathrm{~s} f_{m, Z}{ }^{\prime}$ and $f_{m, X}{ }^{\prime \prime}$, the updated $p d f f_{m X}{ }^{\prime \prime}$ and the 'adjusted' $p d f$ $f_{m, Z}{ }^{*}$ are shown in Figure 3.

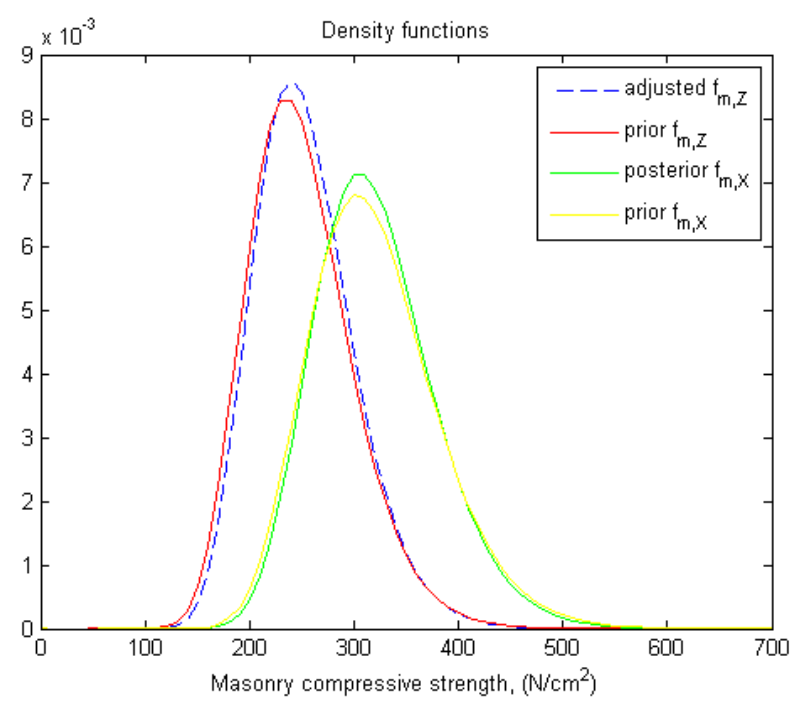

Figure 3: Prior and updated $p d f s$.

\section{Conclusion}

Probabilistic methods and Bayesian updating techniques are often invoked for the reliability assessment of historical buildings. However a probabilistic description of the material properties is affected by great uncertainties, for example due to the 
heterogeneity and to the composite nature of masonry or the wood anisotropy. Moreover, the acquisition of data is also a complicated process, since destructive test may entail the cultural value of the building, so that the only quantitative information available are often represented by the result of destructive test carried out on similar structures.

In this paper, a procedure for the updating of PDF for basic variables based on qualitative judgments and visual inspection is proposed. The procedure is articulated in the following steps:

1. definition of a prior $p d f$ for the relevant mechanical variable pertaining to the structure to be assessed;

2. identification of experimental results obtained on already tested similar and coeval structures;

3. definition of a posterior $p d f$ implementing the aforementioned test results in a Bayesian Updating;

4. definition of a couple of corrective coefficients $a, b$ representing the effect of the Bayesian Updating on the prior $p d f$;

5. application of the corrective coefficients to the parameters of the prior $p d f$ for the structure to be assessed, and definition of suitably 'adjusted' parameters.

According to the described procedure, it is possible to reduce the uncertainty about the considered structure, exploiting Bayesian Analysis carried out on similar and coeval structures. In this way, a more precise probabilistic description of the material characteristics can be achieved without performing any destructive tests on the considered structure, and a more realistic reliability assessment can be performed applying probabilistic methods.

\section{Acknowledgement}

This study has been carried out in the framework of the Leonardo Da Vinci Project CZ/13/LLP-LdV/TOI/134014: "Innovation Transfer in Risk Assessment and Management of Aging Infrastructures".

\section{References}

[1] Mainstone, R., "Reflections on the related histories of construction and design", Proc. of the First International Congress on Construction History, Madrid, pp. 49-60, 2003.

[2] Cabo, J. L. F., "Structure: Size, form and proportion. A historical review on the main criteria for structural design. Theoretical and empirical studies on dead load", Proc. of the First International Congress on Construction History, Madrid, pp. 883-894, 2003.

[3] EN 1990:2002, Eurocode - Basis of structural design, 2002.

[4] Diamantidis, D. \& Holicky, M., Innovative methods for the assessment of existing structures, Czech Technical University in Prague, Klokner Institute, 2012. 
[5] Diekmann, J., "Past Perfect: Historical Antecedents of Modern Construction Practices.” J. Constr. Eng. Manage. 133, 50th Anniversary Special Issue, pp. 652-660, 2007.

[6] Augenti, N., Parisi, F. \& Acconcia E., MADA: online experimental database for mechanical modelling of existing masonry assemblages., Proc. of the 15th World Conference on Earthquake Engineering, Lisbon, Portugal, 2012.

[7] Croce, P. \& Holicky, M., Operational methods for the assessment of existing structures, TEP, Pisa, 2013.

[8] Marsili, F., Croce, P., Muzzi, M., Rosso, E. \& Beconcini, M.L., "Increasing the robustness of the Bayesian Analysis", Proc. of the IABSE Workshop on Safety, Robustness and Condition Assessment of Structures, Helsinki, pp. 194-201, 2015.

[9] Binda, L. \& Saisi, A., "Knowledge of the building, on site investigation and connected problems", Eurocode 8 Perspectives from the Italian Standpoint Workshop, pp. 213-224, 2009.

[10] NTC 2008, Norme Tecniche per le Costruzioni, Roma, 2008.

[11] CNR-DT 212/2013, Istruzioni per la Valutazione Affidabilistica della Sicurezza Sismica di Edifici Esistenti, Roma, 2014.

[12] Aitchison, J. \& Brown, J.A.C., General Properties of the Lognormal Distribution (Chapter 2), The Lognormal Distribution, Cambridge University Press, London, pp. 7-17, 1963.

[13] Magenes, G., Penna, A., Galasco, A. \& Rota M., "Experimental characterisation of stone masonry mechanical properties", Proc. of the 8th International Masonry Conference, Dresda, pp. 247-256, 2010. 